characteristics of autoimmune diseases associated with sarcoidosis (sarcoidosisoverlap group) patients and isolated sarcoidosis (isolated sarcoidosis group) were analyzed and compared.

Results: Autoimmune disease was detected in $15(11.5 \%)$ of 131 patients with sarcoidosis (1Sjögren syndrome, 3rheumatoid arthritis, 1Still disease, 1scleroderma, 4ankylosing spondylitis, 1familial Mediterranean fever, 1gut arthritis, 1immune trombocytopenic purpura, 1 Hashimoto thyroiditis and 1Graves disease). Most of these diseases occurred before (such as RA, AS, Still, FMF) and others after sarcoidosis diagnosis. Among 15 sarcoidosis patients with autoimmune disease 10 were female and 5 were male, the mean age was 50.8 years and mean disease duration was 3 months (1-30 months). When compared with isolated sarcoidosis patients, more hand finger joint involvement, RF positivity, higher ESR and less NSAIDs usage were found in patients with sarcoidosis-overlap group $(p=0.035, p=0.049, p=0.015, p=0.018$ respectively). There was no statistically significant differences between the two groups when evaluated for demographic, clinical parameters and other treatment modalities.

Conclusions: Concomitant autoimmune diseases in patients with sarcoidosis may be often seen. This patients are characterized with more hand finger joint involvement, RF positivity, higher ESR and less NSAIDs usage. Therefore, in patients with a diagnosis of sarcoidosis, it is necessary for the physician to be careful and to make a wider differential diagnosis in terms of the presence of another underlying autoimmune disease. Multicenter, prospective studies involving large numbers of patients are needed to understand whether the association of sarcoidosis-autoimmune diseases is based only on coincidence or on a common etiopathogenesis.

Disclosure of Interest: None declared

DOI: 10.1136/annrheumdis-2017-eular.1376

\section{THU0574 CLASSICAL IMMUNOSUPPRESSION AND DAMAGE PROGRESSION IN A GROUP OF PATIENTS DIAGNOSED WITH BEHCET'S DISEASE}

S. Daia-lliescu, C. Buzatu, A. Borangiu, I. Saulescu, L. Groseanu, V. Bojinca, A. Balanescu, D. Predeteanu, R. Ionescu, D. Opris-Belinski. Internal Medicine and Rheumatology, Sf. Maria Clinical Hospital, Bucharest, Romania

Background: Behcet's Disease is a rare type of vasculitis that involves both arterial and venous blood vessels of all sizes. The type of organ involvement and overall disease activity evaluated in the clinical practice determine the course of treatment and the decision to initiate immunosuppression. Activity scores such as Birmingham Vasculitis Activity score (BVASv3), Behcet's Disease Current Activity Form2006 (BDCAF), or damage indices like Vasculitis Damage Index (VDI) have been developed in this respect.

Objectives: To evaluate the ability of classical immunosuppressant therapy to prevent damage progression. To find the correlation between disease activity scores: BVASv3, BDCAF, long term treatment, immunosuppressant use and damage after remission, as calculated by VDI.

Methods: A study on a cohort of patients diagnosed with Behcet's Disease from an Internal Medicine and Rheumatology Clinic was performed. Activity and damage scores,BVASv3,BDCAF and VDI after obtained remission, were calculated. The documented cases were diagnosed according to the International Criteria for Behçet's Disease (ICBD).Windows Excel/SPSS20.0 (Spearman's correlation)were used to analyse the data.

Results: The study included 16 patients treated with long term cortisone and immunosuppressive therapy. The mean age at the time of the diagnosis was $32.3 y e a r s$ with a male predominance $62 \%$ (10 patients). Severe systemic involvement was present in 10 cases (Ophthalmological involvement-6cases, recurrent venous thrombosis-6cases, pulmonary vasculitis- 1 case, severe cardiac involvement-1case, central nervous system involvement-3cases) and all patients received classical immunosuppression (cyclophosphamide, azathioprine). The mean scores for BVASv3 and BDCAF at the time of the diagnosis were 9 and 4.12.A strong correlation was identified between BVASv3 and BDCAF $(r=0.830, p<0,001)$. The use of immunosuppressive therapy due to severe organ involvement and long-term immunosuppression correlated stronger with BVASv3 $(r=0.718)$ than with BDCAF $(r=0.533)$. Vasculitis damage index (VDI) calculated after remission was obtained. There was an important correlation between disease activity scores and damage (BVASv3-VDI $r=0,687, p<0,001$, BDCAF-VDI $r=0,676, p<0,001)$. Types of treatment were evaluated, a comparison was made between long-term cortisone therapy and immunosuppression. There was a stronger correlation between long term cortisone use and VDI $(r=0,600)$ than between immunosuppression duration and damage $(r=0,472)$.

Conclusions: Damage progression is influenced by disease activity, as calculated by activity scores (BVASv3 and BDCAF). Classical immunosuppression is used for severe organ involvement and for limiting new organ lesions once started. There was a stronger correlation between long-term cortisone use and VDI than between immunosuppression duration and damage. The damage index increased by irreversible organ damage due to disease activity and long term cortisone use, but not due to the immunosuppressive therapy.

References:

[1] DavatchiF,et al How to deal with Behcet's disease in daily practice. Int $\mathrm{J}$ Rheum Dis. 2010 May.

[2] Raashid Ahmed Luqmani Disease assessment in systemic vasculitis. Nephrol Dial Transplant 2015.
Acknowledgements: The first two authors,Sinziana Daia-lliescu and Casandra Buzatu contributed equally to this study.

Disclosure of Interest: None declared

DOI: 10.1136/annrheumdis-2017-eular.5530

\section{THU0575 EFFICACY OF RITUXIMAB IN RESISTANT PALINDROMIC RHEUMATISM:FIRST REPORT IN LITERATURE}

S. Sreenath ${ }^{1}$, S. Cherian ${ }^{1}$, G. Antony ${ }^{1}$, U. Mony ${ }^{2}$, P. Shenoy ${ }^{1} \cdot{ }^{1}$ Centre for Arthritis and Rheumatism (CARE); ${ }^{2}$ Molecular Medicine, AIMS, Cochin, India

Background: Palindromic rheumatism (PR) although often considered as a benign disease can be severe and resistant to DMARDs in some patients. In these patients it can result in almost daily attacks, migrating from joint to joint resulting in poor quality of life. Rituximab has been proven to be effective in treatment of seropositive RA.

Objectives: To determine the efficacy and safety of Rituximab in patients with seropositive PR who had an inadequate response to CsDMARDs

Methods: PR was diagnosed based on criteria proposed by Hannonen $P$ et al. Seropositive (ACPA \pm RF positivity) PR patients who had active disease despite being treated with two Cs DMARDs for $>3$ months, were treated with Rituximab. Active disease was defined as $>4$ attacks per month requiring intake of NSAIDS. All the patients were started on $500 \mathrm{mg}$ of rituximab after baseline work up. If complete control of palindromic attacks was not achieved and B cells were detectable in the peripheral blood by flow cytometry another $500 \mathrm{mg}$ infusion was given after 2 weeks. Patients were continued on maximum tolerable dose of DMARDS. Patients were given repeat infusion of Rituximab once the patient developed clinical relapses as evidenced by recurrence of palindromic attacks.

Results: Twenty three patients with a mean age of $44.60 \pm 13.51$ yrs and mean disease duration of $5.47 \pm 3.25$ yrs were included. All patients were positive for ACPA while 17 patients were positive for RF. These patients were on a background of minimum of 2 DMARDs. Despite the maximum tolerable dose of DMARDs they had mean attack rate of $5.30 \pm 2.38$ attacks per month. During a mean follow up of $14.17 \pm 8.62$ months seven patients required two infusions and three patients required three infusions. Of the 33 infusions $500 \mathrm{mg}$ was effective in controlling the attacks majority ( $88 \%)$ of the times. Seven patients required another 500 mg infusion after 2 weeks as initial $500 \mathrm{mg}$ dose failed to achieve complete control of disease and B cell were not depleted in the peripheral blood. At one month follow up all patients achieved complete control of disease. Remission lasted for $10.33 \pm 5.75$ months. When symptoms recurred patients were treated with rituximab again and all regained complete control of the symptoms. None of the patients evolved into RA during the study period. No serious adverse events were observed. Five patients experienced minor allergic reactions during infusion which were managed according to the standard protocol.

Conclusions: This case series indicates in patients of PR resistant to Cs DMARDs rituximab not only achieves disease control but also prevents progression to RA. To best of our knowledge this is the first report regarding efficacy of rituximab in PR. Although it needs to be proved in a larger blinded RCT this early data indicates that Rituximab may be a therapeutic option to prevent development of $\mathrm{RA}$ in seropositive patients.

References:

[1] Hannonen P, Möttönen T, Oka M. Palindromic rheumatism. A clinical surveyof sixty patients. Scand J Rheumatol. 1987;16(6):413-420.

[2] Sanmarti R, Cañete JD, Salvador G. Palindromic rheumatism and other relapsing arthritis. Best Pract Res ClinRheumatol. 2004;18(5):647-661.

Disclosure of Interest: None declared

DOI: 10.1136/annrheumdis-2017-eular.5723

\section{THU0576 THERAPEUTIC OPTIONS FOR PATIENTS WITH RARE RHEUMATIC DISEASES: A SYSTEMATIC REVIEW AND META-ANALYSIS}

T.T.A. Bender $^{1}$, M. Mücke ${ }^{1,2,3}$, J. Leyens ${ }^{1}$, C. Stieber ${ }^{1,4}$, D. Kravchenko ${ }^{5}$ M.F. Seidel 6,7 . ${ }^{1}$ Center for Rare Diseases Bonn (ZSEB): ${ }^{2}$ Institute of General Practice and Family Medicine; ${ }^{3}$ Department of Palliative Medicine; ${ }^{4}$ Institute of Human Genetics; ${ }^{5}$ University Hospital Bonn, Bonn, Germany; ${ }^{6}$ Department of Oncology, Hematology and Rheumatology, University Hospital Bonn, Bonn, Germany; ${ }^{7}$ Schmerzklinik Basel, Basel, Switzerland

Background: Rare rheumatic diseases are challenging for both patients and clinicians. This problem is further propelled by the scarce number of approved treatment regimens. Therapy is often limited to immunosuppression with corticosteroids or off-label use of drugs for common rheumatic diseases.

Objectives: We have recently described a set of 82 classified rare diseases in rheumatology [1]. In this systematic review, we analysed the evidence for therapeutic regimens from randomized clinical trials of rare rheumatic diseases.

Methods: For this systematic review and meta-analysis, we searched the Cochrane Central Register of Controlled Trials (CENTRAL), MEDLINE, PUBMED and SCOPUS, up to 20th of October 2016. To validate the search strategy, we selected sentinel references.

We included randomized controlled trials regarding rare rheumatic diseases and put a focus on pharmacological treatment compared with placebo, application of 
two drugs, or standard therapy. The term "rare" was defined by the European Union as a condition that occurs in no more than 1 in 2,000 individuals.

Two review authors independently assessed trial quality and extracted the data. We screened the search results and included studies if they met the selection criteria. If we identified two or more trials that investigated the same rare disease and used the same assessment tools we performed a meta-analysis.

Results: 135 studies were screened, of which 34 met the inclusion criteria. In total, we analysed data on 11 different orphan diseases, encompassing 2,324 participants. There was a high degree of statistical and clinical heterogeneity in these trials. Several sources of potential bias were identified in the included studies, for example, a lack of description of the blinding methods and allocation concealment, as well as the small size of the study populations. We included studies such as rituximab against cyclophosphamide in ANCA-associated vasculitis. These studies demonstrated a non-inferiority of rituximab. The meta-analysis resulted a combined odds ratio $(\mathrm{OR})$ of 1.42 in favour of rituximab $(95 \% \mathrm{Cl})$. Further metaanalyses were possible for another 22 studies involving, among others, Behçet's disease, systemic sclerosis, cryopyrin-associated periodic syndromes, and giant cell arteritis. Compounds studied were immunosuppressants like corticosteroids, methotrexate and azathioprine, or biologicals such as rilonacept, infliximab, and canakinumab.

Conclusions: A high degree of evidence is hampered by the limited number of study participants in each trial. On the other hand, diseases such as systemic sclerosis, ANCA-associated vasculitides, or Behçet's disease had more high quality trials available. The amount of data for most other rare disease remains unsatisfactory.

\section{References:}

[1] Leyens J, Stieber C, Bender TTA, Mücke M, Seidel MF. (2016) Classification of rare diseases in rheumatology demonstrates a combined prevalence double to the prevalence of ankylosing spondylitis. Ann. Rheum. Dis. 75(Suppl2): 618.

Disclosure of Interest: None declared

DOI: 10.1136/annrheumdis-2017-eular.2946

\section{THU0577 TOCILIZUMAB MONOTHERAPY FOR ADULT ONSET STILL'S DISEASE - RESULTS OF 52-WEEK TREATMENT OF A PROSPECTIVE, SINGLE-CENTER, SINGLE-ARM, OPEN TRIAL}

T. Kondo, Y. Okada, A. Shibata, K. Chino, T. Kurasawa, A. Okuyama, H. Takei, K. Amano. Department of Rheumatology and Clinical Immunology, Saitama Medical Center, Saitama Medical University, Kawagoe, Saitama, Japan

Background: Adult-onset Still's disease (AOSD) is a systemic inflammatory disease of unknown etiology. Corticosteroids still provide mainstay AOSD therapy despite various adverse effects. Recently, AOSD patients have been successfully treated with anti-cytokine therapies such as with TNF- $\alpha$ blocking agents, an IL-1 receptor antagonist, and an anti-IL-6 receptor monoclonal antibody. Among these case reports, TCZ seems to be highly effective for treating patients refractory to TNF antagonists and IL-1 antagonist.

Objectives: To assess the efficacy and safety of tocilizumab (TCZ) monotherapy for the induction therapy of adult onset Still's disease (AOSD) in a prospective single-arm, single-center, cohort, pilot study.

Methods: Seven AOSD patients (male 2, female 5) who had agreed with our prospective trial since April 2010 till May 2015 were enrolled. Our study protocol is that patients received $8 \mathrm{mg} / \mathrm{kg}$ of intravenous TCZ fortnightly for the first two months (five courses), then monthly for the next 5 months and after that they stop TCZ therapy and we monitor symptoms about AOSD relapses. In this report, we evaluated the efficacy and safety in 52 week. Efficacy was evaluated by serum markers (WBC, CRP and serum ferritin), clinical symptoms and ratio of patients who required additional therapy, and safety was evaluated by adverse events for 52 week.

Results: The mean age was 41.4. The ratio of fever, arthralgia, rash and sore throat are $100 \%(n=7 / 7), 100 \%(n=7 / 7), 85.7 \%(n=6 / 7)$ and $71.4 \%(n=5 / 7)$ respectively. LOCF analysis revealed that WBC, CRP and serum ferritin level decreased significantly from $14757 \pm 4667 / \mu \mathrm{l}$ to $6985 \pm 2903 / \mu \mathrm{l}$, from $13.4 \pm 7.1 \mathrm{mg} / \mathrm{dl}$ to $0.3 \pm 0.6 \mathrm{mg} / \mathrm{dl}$ and from $6927 \pm 5376 \mathrm{ng} / \mathrm{ml}$ to $2416 \pm 5589 \mathrm{ng} / \mathrm{ml}$ at month 7 (TCZ final infusion) respectively (each, $\mathrm{P}<0.01)$. The improvement rate of fever, arthralgia and eruption were 100\% $(n=7 / 7), 85.7 \%(n=6 / 7)$ and $85.7 \%(n=6 / 7)$ respectively at month 7 . One patient couldn't continue TCZ therapies because of lack of efficacy $(14.3 \%, n=1)$ and required additional therapy (prednisolone) at

Change of Symptom Prevalence

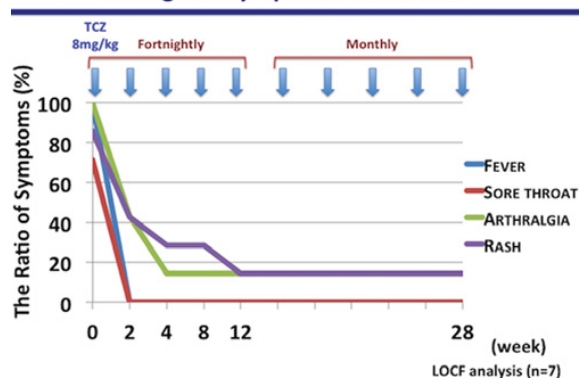

week 2. Another patient also abandoned this trial due to adverse event $(14.3 \%$, $\mathrm{n}=1$, urinary tract infection). The other 5 patients could complete the 7-month course of the study and had no symptoms at month 7. Four of five patients had no flare-up signs until month 5 after stopping TCZ. One patient had relapse symptoms such as rash and arthralgia and increased serum level of CRP and serum ferritin at month 2 after final TCZ infusion. There were no serious adverse events during the course of the trial.

Conclusions: TCZ monotherapy can be an alternative treatment strategy for AOSD in some patients.

References:

[1] Sakai R, et al.:Clin Rheumatol. 2012 Mar;31(3):569-74.

[2] Ortiz-Sanjuán F et al.: Arthritis Rheumatol. 2014 Jun;66(6):1659-65.

Disclosure of Interest: T. Kondo: None declared, Y. Okada: None declared, A. Shibata: None declared, K. Chino: None declared, T. Kurasawa: None declared, A. Okuyama: None declared, H. Takei: None declared, K. Amano Grant/research support from: Chugai Pharmaceutical Co.Ltd.

DOI: 10.1136/annrheumdis-2017-eular.4922

\section{THU0578 SPECTRUM OF THE DISEASES WITH ORBITAL INVOLVEMENT IN RHEUMATOLOGY: SINGLE-CENTER STUDY}

V. Vasilyev ${ }^{1}$, V. Gorodetskiy ${ }^{1}$, T. Safonova ${ }^{2}$, N. Probatova ${ }^{3}$, N. Kokosadze ${ }^{3}$, A. Pavlovskaya ${ }^{3}$, A. Kovrigina ${ }^{4}$, I. Gaiduk ${ }^{5}$. ${ }^{1}$ Nasonova Research Institute of Rheumatology; ${ }^{2}$ Research Institute of Ophthalmic diseases; ${ }^{3}$ N.N. Blochin Russian Cancer Research Center; ${ }^{4}$ Hematology Research Center; ${ }^{5}$ A.I. Evdokimov Moscow State Dental University, Moscow, Russian Federation

Background: It is assumed that in ophthalmological clinical practice lymphoproliferative disorders in patients with affection of different orbital organs and tissues constitute $25-55 \%$ and $21 \%$ of patients have lgG4-related ophthalmic disease $[1,2]$.

Objectives: To evaluate the most common conditions affecting the orbit in rheumatologic clinical practice.

Methods: During 2004 - 2016 years 138 patients (male - 33, female - 105) with eyelid edema and/or tumefactive lesions in the orbit were examined in Nasonova Research Institute of Rheumatology. In all patients full clinical, ophthalmological, serological (rheumatoid factor, C-reactive protein, $\lg$, $\lg G 4, \lg M, \lg A$, ANA, anti-Ro/La, C3/C4 complement) examination was carried. In all cases diagnosis was verified pathomorphologicaly with immunohystochemical staining (anti-CD 138, CD 68, IgG, IgG4, $\kappa$-chain, $\lambda$-chain). Diagnosis was established on the basis of pathomorphological examination of different tissues: orbit -79 patients, parotid salivary gland -40 , submandibular salivary gland -14 , nasal -4 , lymph nodes 3 and other -6 . In 79 cases (54\%) the diagnosis was established on the results of orbital biopsy.

Results: Different non-neoplastic diseases were diagnosed in 108 patients $(78.5 \%)$ and 30 had different conditions of hematological spectrum, including malignant conditions in $25(18.2 \%)$ patients (see table 1). Some patients at baseline had simultaneous involvement of the major salivary glands ( 23 patients with IgG4-related disease, 35 with sarcoidosis, 12 with non-Hodgkin lymphomas and 2 with AL-amyloidosis). The most rare conditions affecting the orbit were Cogan's syndrome, relapsing polychondritis, Erdheim-Chester disease, NK/T-cell nasal lymphoma and calcifying aponeurotic fibroma.

Table 1. Spectrum of the diseases with orbital involvement in rheumatologic practice $(n=138)$

\begin{tabular}{|c|c|c|c|c|c|}
\hline & $\begin{array}{l}\mathrm{N}, \\
\text { pts }\end{array}$ & $\mathrm{N}, \%$ & & $\begin{array}{l}\mathrm{N}, \\
\text { pts }\end{array}$ & $\mathrm{N}, \%$ \\
\hline Non-neoplastic conditions & 108 & 78.5 & Hematologic conditions & 30 & 21.5 \\
\hline - IgG4-related disease & 48 & 35.0 & $\begin{array}{l}\text { non-Hodgkin } \\
\text { lymphomas }\end{array}$ & 20 & 14.5 \\
\hline $\begin{array}{l}\text { - Granulematous } \\
\text { lesions (sarcoidosis, } \\
\text { granulematosis with } \\
\text { polyangiitis, } \\
\text { necrosing sarcoidal } \\
\text { granulematosis) }\end{array}$ & 41 & 29 & $\begin{array}{l}\text { - Erdheim-Chester } \\
\text { disease }\end{array}$ & 1 & 0.7 \\
\hline $\begin{array}{l}\text { Autoimmune } \\
\text { dacryoadenitis (non- } \\
\text { differentiated) }\end{array}$ & 7 & 5 & - $\mathrm{AL}$-amyloidosis & 3 & 2.1 \\
\hline $\begin{array}{l}\text { Idiopathic orbital } \\
\text { inflammation }\end{array}$ & 7 & 5 & $\begin{array}{l}\text { - NK/T-cell nasal } \\
\text { lymphoma }\end{array}$ & 1 & 0.7 \\
\hline $\begin{array}{l}\text { Endocrin } \\
\text { ophthalmopathy }\end{array}$ & 2 & 1.4 & - Histiocytosis & 4 & 2.9 \\
\hline - Cogan's syndrome & 1 & 0.7 & $\begin{array}{l}\text { - Cal cifying } \\
\text { aponeurotic fibr oma }\end{array}$ & 1 & 0.7 \\
\hline $\begin{array}{l}\text { - Relapsing } \\
\text { polychondritis }\end{array}$ & 1 & 0.7 & & & \\
\hline
\end{tabular}

Conclusions: In rheumatologic practice in $78.5 \%$ of patients with orbital involvement different non-neoplastic conditions are diagnosed: IgG4-related ophthalmic disease (35.0\%), granulematous lesions (29\%). The most common hematological disorders in rheumatologic clinic are non-Hodgkin lymphomas $(17.5 \%)$ and histiocytosis $(3.5 \%)$

References:

[1] Japanese study group of IgG4-related ophthalmic desease. A prevalence 